Meta

Journal des tradlucteurs

Translators' Journal

\title{
Théories de la traduction (1989), Tunis, Fondation nationale
} Carthage, $290 \mathrm{p}$.

\section{Béchir Ouerhani, Saïd Mosbah, Sayda Sandly et Lassaâd Oueslati}

Volume 45, numéro 3, septembre 2000

La traduction dans le monde arabe

URI : https://id.erudit.org/iderudit/003774ar

DOI : https://doi.org/10.7202/003774ar

Aller au sommaire du numéro

Éditeur(s)

Les Presses de l'Université de Montréal

ISSN

0026-0452 (imprimé)

1492-1421 (numérique)

Découvrir la revue

Citer ce compte rendu

Ouerhani, B., Mosbah, S., Sandly, S. \& Oueslati, L. (2000). Compte rendu de

[Théories de la traduction (1989), Tunis, Fondation nationale Carthage, 290 p.]

Meta, 45(3), 561-562. https://doi.org/10.7202/003774ar 


\section{DOCUMENTATION}

\section{Comptes rendus}

Théories de la traduction (1989), Tunis, Fondation nationale Carthage, 290 p.

Il s'agit d'un ouvrage collectif qui regroupe six articles réalisés dans le cadre du groupe de recherche de Beït Al-Hikma, Fondation nationale pour la traduction, l'établissement des textes et les études.

Les six textes pourraient être regroupés comme suit:

- les textes de M. Mensya et de K. Gaha sont nettement d'orientation historique;

- les textes de M. Jazzar, de B. Gamarti et de A. Y. Marzouki privilégient la problématique des types de traduction scientifique et littéraire;

- celui de M. Agina situe la réflexion au niveau des techniques de la traduction.

Aussi paradoxal que cela puisse paraître, le titre dans sa version bilingue - en arabe La traduction et ses théories (littéralement), en français Théories de la traduction - trahit une ambiguïté lourde de conséquences. Le titre français suggère que l'ouvrage renferme une présentation critique de théories, anciennes et modernes, en rapport avec la traduction; le titre arabe, bien qu'il garde la même configuration, dissocie les deux dimensions.

En effet, cette ambiguïté structure tout l'ouvrage:

1. Animé d'une préoccupation historique, Mensya fait un exposé détaillé sur la traduction dans le monde arabo-musulman des $\mathrm{VI}^{\mathrm{e}}$ au $\mathrm{IX}^{\mathrm{e}}$ siècles en précisant que l'échange entre l'Orient et l'Occident dans ce domaine, contrairement à ce qu'on croit, est antérieur à l'avènement de l'Islam. Quant à lui, Gaha s'est intéressé particulièrement à la renaissance arabe ( $\mathrm{XIX}^{\mathrm{e}}$ siècle) en défendant la thèse que la traduction chez les Arabes est étroitement liée aux périodes d'essor parce qu'ils sont convaincus de son rôle déterminant dans le développement général de la société; selon lui, cet essor aurait avorté à cause de la colonisation.

2. Les considérations historiques servant de toile de fond aux trois contributions consacrées aux types de traduction pèsent sur les objectifs que se sont fixés les auteurs de ces textes. En effet, celui de Marzouki est axé fondamentalement sur des données de nature socioculturelle, ceux de Gamarti et de Jazzar respectivement sur les spécificités des textes scientifiques et des textes littéraires, le tout étant coulé dans une matrice didactique.

3. L'exposé d'Agina se distingue par des considérations générales relatives aux contraintes objectives imposées par le passage d'un système linguistique à un autre.

Cet ouvrage, qui renferme des données historiques très précieuses pour tous ceux qui ignorent l'importance de la traduction dans la tradition arabo-musulmane, ne répond pas aux attentes suggérées par le titre, ce qui aurait pu être évité si on l'avait intitulé: La traduction arabe: histoire et préoccupations actuelles. 
Il n'en demeure pas moins qu'il représente un outil de travail très utile pour les étudiants en maîtrise de traduction.

BÉchir OUERHANi

SAÏD Mosbah

SAYda SANDLY

LASSAÂd OUESLATI 